Training, and identifying and maintaining standards for its delivery, are key functions of the Royal College of Psychiatrists. Over the past 30 years the College can look at its record with pride: it has set standards that are fairly similar across different countries and has done so with a mixture of gentle persuasion, approbation and warning of the likely outcome of losing accreditation. This has been a useful strategy in establishing standards and ensuring that they are met. However, changes are now necessary for a number of reasons. Psychiatric services have changed beyond recognition. Mental asylums have been replaced with community-based mental health centres and different types of acute services such as home treatment and assertive outreach. The role of the consultant has also changed and revalidation and reaccreditation are likely to play a significant part in the personal development of the next generation of consultants. Advances in information technology have also meant that a changing set of skills has become necessary for delivery of services in the 21st century.

\section{Generators of change}

Impetus for change in the College's training structures has come from three directions. First, the introduction of the European Working Time Directive has meant that traditional methods of delivering training are no longer appropriate. Second, the Modernising Medical Careers project (http://www.mmc.nhs.uk/ pages/home) has introduced a second year of foundation training, ostensibly to give trainees exposure to a larger number of specialties and consequently a wider career choice. The third and single most important change, which will affect all the medical Royal Colleges, has been the establishment of the national Postgraduate Medical Education and Training Board (PMETB), charged with monitoring and maintaining standards of postgraduate medical education.

\section{The Postgraduate Medical Education and Training Board}

Established 2 years ago, PMETB 'went live' on 30 September 2005. It replaces the Specialist Training Authority (STA) and the Joint Committee for General Practice Training (JCPTGP). One of the primary tasks of the Board is to ensure that examinations are fit for purpose. The Board is also responsible for ensuring that assessments of doctors in training are curriculum-based, fair, reliable, feasible and costeffective. The purpose of each assessment must be specified and made available to all stakeholders, and the level and sequence of assessments along a career pathway must be laid out. Feedback must be given and any weaknesses in the trainees's performance must be addressed in further training. Assessment methods must be transparent and in the public domain. Lay assessors must be involved in the development of assessment.

\section{Changes within the College}

The Royal College of Psychiatrists' response to change has been sustained and appropriate. The two forthcoming changes that will have greatest effect on the College are the introduction of a single unified training grade and PMETB's regulation of assessment methods and its inspection of training schemes.

In the autumn of 2007, the current basic and higher specialist grades will be replaced with a single grade covering the full 6 years of training. Consequently, training scheme organisers, postgraduate deans and directors of medical education are being encouraged not to offer long-term contracts to trainees at the present time.

Owing to the requirements of Modernising Medical Careers and the European Working Time Directive, training and its delivery are likely to become modular. The first $2 \frac{1}{2}$ years of the 6-year

Dinesh Bhugra is Dean of the Royal College of Psychiatrists, Professor of Mental Health and Cultural Diversity, and head of the Section of Cultural Psychiatry at the Institute of Psychiatry, London (Box 25, Health Services Research Department, Institute of Psychiatry, King's College London, De Crespigny Park, London SE5 8AF, UK. Email: d.bhugra@iop.kcl.ac.uk). His research interests are in cultural psychiatry, sexual dysfunction, medical education and primary care psychiatry. 
training will provide exposure to a range of subspecialties, much as basic training currently does. During the remaining 31/2 years trainees will focus on their chosen specialty, as in current higher training. Accordingly, training for the College's membership examinations (the MRCPsych) will be modular and trainees will be expected to study a number of modules prior to assessment. PMETB is looking into clear entry criteria for all specialties. There will be a knowledge-based assessment, probably at the end of the first year of specialist training in psychiatry. A clinical (possibly OSCE - objective structured clinical examination) assessment may be conducted before entry into sub-specialty training. These will be roughly equivalent to the current parts I and II of the MRCPsych examination.

\section{The new curriculum}

The College has sent its new curriculum for specialist training to PMETB for its informal approval. The framework of the curriculum and its principles have been developed from those underpinning the existing competency-based higher specialist training, with additional features to meet the PMETB's requirements. For our trainees, the focus of learning and of assessments will be the workplace. For our trainers and assessors, the foci of the curriculum and assessment will be performance (what the trainee actually does) and competence (ability successfully to perform a task or procedure). Much more will be expected than the simple recall of knowledge. There is likely to be an end-of-training assessment based on a portfolio, interview and competency-led assessments. There might also be a number of workplacebased assessments, which might include a long case assessment, a number of mini clinical examinations and case-based orals together with assessment of communication and liaison skills. Trainees will have to maintain a portfolio recording acquisition of competencies. Workplace-based assessments would be conducted by educational supervisors rather than by clinical supervisors. The changes in the MRCPsych will focus on fitness of purpose and ensuring that knowledge and competencies are appropriately assessed. After March 2006, PMETB will approve all training schemes.

\section{Potential disadvantages of the training revisions}

The changes to medical training may pose some problems for psychiatry and the College. For example, if there are insufficient posts in foundation year 2, smaller specialties such as psychiatry may suffer because potential trainees may not have had exposure to them and they may miss out on those who have not made up their minds. The single training grade may make it difficult for those who find that they are not suited to psychiatry to switch to another specialty, as each medical Royal College will be expected to have strict entry criteria to its specialties. The proposed changes also have major resource implications that require urgent discussion. It is possible that the role of the medical Royal Colleges will have to alter and that they will have to deliver changes over which they have limited control.

Some of the changes may result in better delivery of training and, eventually, in service development. However, if not carefully monitored and properly resourced, the proposed 'improvements' may land the medical profession in a bigger mess.

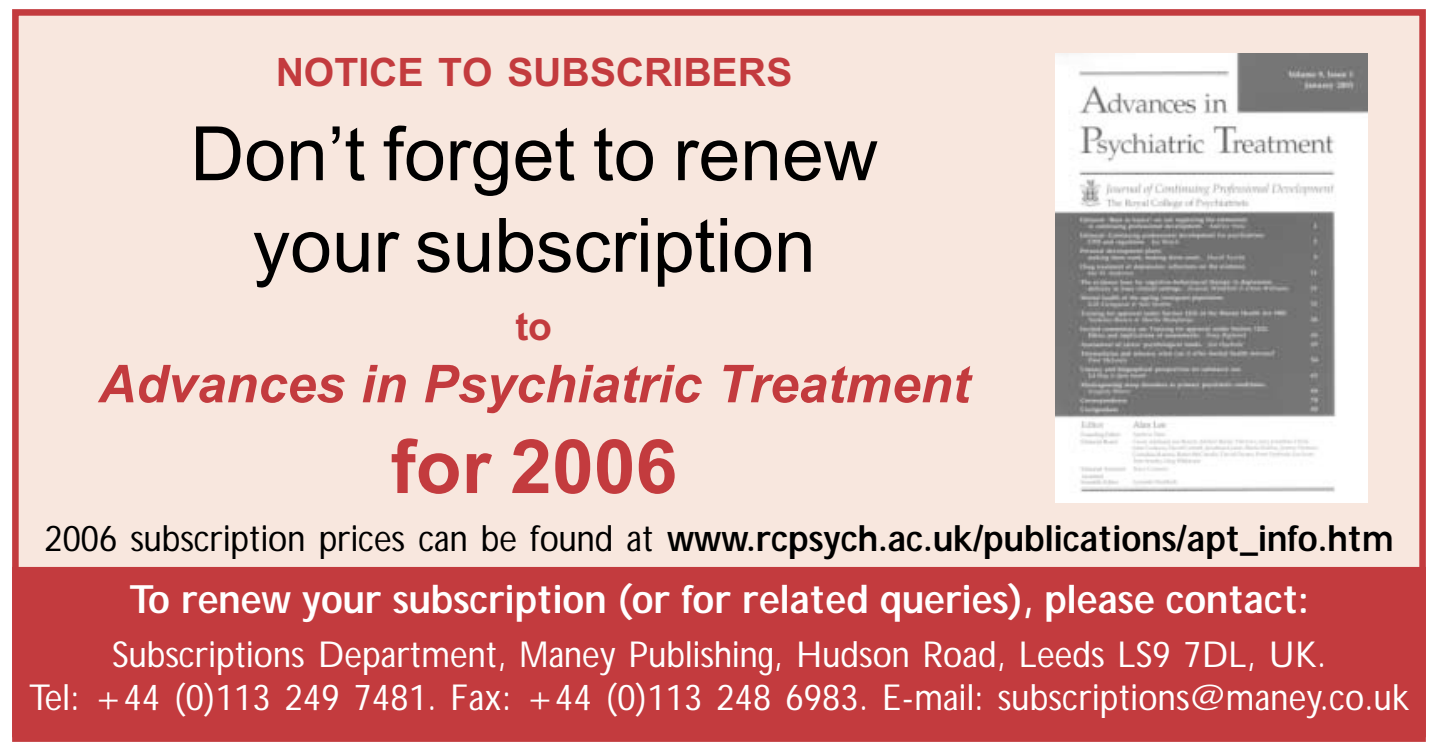

